\title{
DITHERING ALGORITHMS FOR VARIABLE DOT SIZE PRINTERS
}

\author{
V. Ostromoukhov, P. Emmel, N. Rudaz, I. Amidror, R.D. Hersch \\ Ecole Polytechnique Fédérale, Lausanne, Switzerland \\ hersch@di.epfl.ch
}

\begin{abstract}
Dither-based methods for the halftoning of images on multi-level printing devices such as multi-level inkjet printers are presented. Due to the relatively large size of single droplets, halftoning algorithms are still needed. However, since halftoning occurs between the basic levels attainable by printing one, two or several droplets at the same position, artefacts are less visible than in equal resolution bilevel printers. When dithering algorithms are used for the halftoning task, the dither threshold tiles should have oblique orientations so as to make the halftoning artifacts less visible. They should be designed so as to break up the inherent artifacts of variable dot size printers, such as for example continuous lines made up of elongated elliptic dots. The resulting visual effects are shown by simulating the printed dots of a multilevel inkjet printer.
\end{abstract}

\section{INTRODUCTION}

In the last two years, 300 dpi to $600 \mathrm{dpi}$ high-quality ink jet printers have been offered for desktop publishing at very low cost (below 300 dollars). New halftoning algorithms based on dispersed-dot dithering [1], [2], on improved error-diffusion schemes [3] or on combinations of error-diffusion and dithering techniques [4] have provided the means to reproduce both grayscale and colour images.

Currently, ink-jet device manufacturers are making efforts to put on the market low-cost variable dot size ink-jet printers able to reproduce multiple intensity levels. Multi-level inkjet printers seem easier and cheaper to develop than devices having a significantly higher resolution. The main effort resides in ensuring a constant, repetitive small droplet diameter and at the same time a minimal dot gain by minimizing the ink spread on paper.

Multiple intensity levels per pixel are achieved by printing one, two or several droplets at the same position. Since however a single droplet has a minimal diameter, say $50 \%$ of the diameter of the largest printable dot size, the first darkness level (or surface coverage level) an ink-jet printing device may print is at least $25 \%$, the second darkness level is at least $45 \%$, and the remaining levels cover the darker levels between $45 \%$ and $100 \%$ darkness. It is therefore of capital importance to use halftoning algorithms in order to obtain additional intermediate intensity levels (Fig. 1), e.g. levels between 0 and $25 \%$ darkness, levels between $25 \%$ darkness and $45 \%$ darkness, etc.

The quality criteria for judging and comparing halftoning algorithms are the following:

- visibility of individual dots or screen elements should be minimized

- the number of intensity levels should be large enough (> 40) to avoid banding effects
- structure artifacts, i.e. repetitive or semi-repetitive visible structures should be avoided

- false contours due to sharp halftone structure changes should be avoided

This paper presents dither-based methods for the halftoning of images on multi-level printing devices. The resulting visual effects are shown by simulating the printed dots of a multi-level inkjet printer.

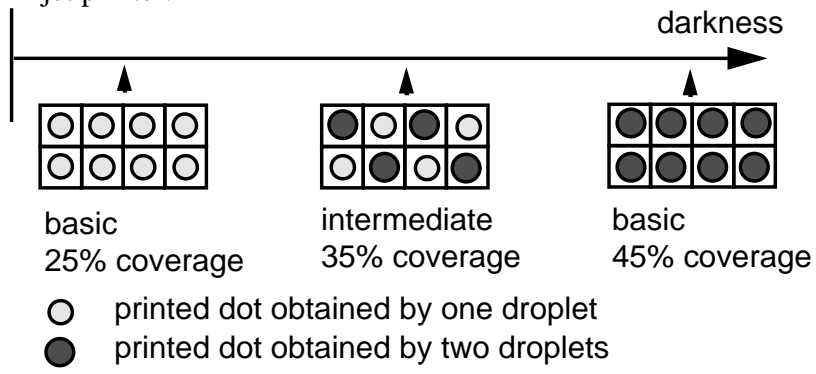

Figure 1. Intermediate darkness levels obtained by dithering between basic levels

\section{DITHER TILE BASED MULTI-LEVEL HALFTONING}

As mentioned in the introduction, multi-level halftoning aims at generating additional intensity levels between the levels produced by printing successive ink drops on paper. When the set of available ink drops produces round dots, and when the colour inks are near to ideal inks or when colour layers can be placed without phase shifts one on top of the other, colour layers can be halftoned separately by a dispersed-dot dithering method such as Bayer dispersed-dot dithering [5], rotated dispersed dither [2] or with the help of any adequate dispersed-dot dither threshold array.

Dither-based halftoning methods [6],[7],[8] are based on dither tiles paving the plane. Parallelogram or respectively hexagonal dither tiles for dispersed-dot dither can be generated recursively with two-fold (Fig. 2a) or respectively three-fold dispersion (Fig. 2b) of threshold levels [9].

The number of additional intensity levels which may be produced between two levels given by $k$ and $k+1$ droplets printed on a single pixel depends on the size of the dither tile. One has to be careful to select a small dither tile, since a large dither tile contains low frequency components and therefore generates more visible artifacts at low and middle resolution. Between the highest intensity level (no droplet printed) and the next lowest intensity level (one droplet printed), the dither array based 
multi-level dithering method behaves in the same way as bi-level dithering and produces similar artifacts, but at a reduced intensity.

At an intermediate level between $k$ and $k+1$ droplets printed on a single pixel, artifacts are still visible, especially for $k=1$ or $k=2$. Figure 6 illustrates the halftones produced between the intensity levels associated with $k=1$ and $k=2$ droplets for a Bayer dither array, a parallelogram dither tile and a hexagonal dither tile. It is immediately apparent that the Bayer dither array generates visually disturbing artifacts such as horizontal and diagonal crosses. Artifacts are less visible with parallelogram and hexagonal dither arrays since they all have an oblique orientation.

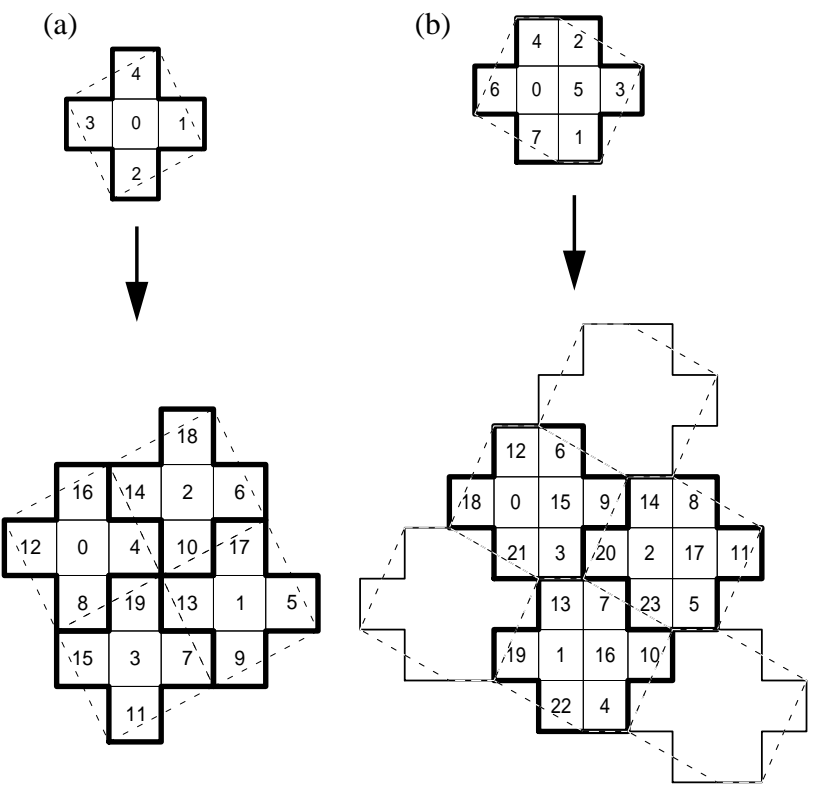

Figure 2. Recursive generation of a parallelogram (a) and hexagonal (b) dispersed dither tiles

In some cases, due to the displacement of the ink-jet head, successive droplets are not printed exactly at the same place, but in a slightly eccentric manner. The resulting printed dot has an elliptic shape. Since the elliptic dot touches neighbouring elliptic dots first in one direction and only after a certain number of intensity levels in the other direction, at certain levels, bands between elliptic dots become visible (Fig. 3a). In order to break these vertical or horizontal bands, the dither threshold levels should be arranged to produce an elliptic dot growing pattern breaking the continuity of the bands (Fig. 3b).

\section{SIMULATION OF REAL IMPACTS}

The circular and elliptic dots shown in Fig. 3 represent only a very coarse approximation of the halftoned intensity levels printed by a multi-level printer. A more accurate approximation, which is closer to the output of a real printer (Fig. 4) can be obtained by varying the position of the centers of individual droplets randomly by a maximum of $\pm 5 \%$ of the circle's diameter in the vertical direction and $\pm 15 \%$ of the circle's diameter in the horizontal direction. In addition, the circle making up the boundary of individual droplets is distorted so as to look like the boundaries of real printed inkjet droplets. We achieve this by defining on the periphery of the circular droplet 20 equidistant points whose locations are varied randomly in $\mathrm{x}$ an $\mathrm{y}$ direction by a maximal amount of $\pm 10 \%$ of the circle's diameter.

The examples of the simulated halftones at level $i+1$ shown in Fig. 5 have been obtained as follows. At halftoning time, when according to the comparison between input intensity level and dither threshold level, the lower basic level needs to be selected, a single droplet is generated on the output grid according to the method described above. When the higher basic level needs to be selected, two droplets are generated, horizontally distant one from another by 0.2 grid units. Centers of the droplets as well as their boundaries are then perturbed by the method described above.

The reduced-size halftone patterns shown in Figure 5 show that both the parallelogram dispersed dither array (Fig. 5b) and the hexagonal dispersed dither array (Fig. 5c) break the horizontal patterns produced by Bayer's dispersed dither method (Fig. 5a).

(a) Bayer Dispersed Dither level i+2

(b) Parallelogram Dispersed Dither level i+2

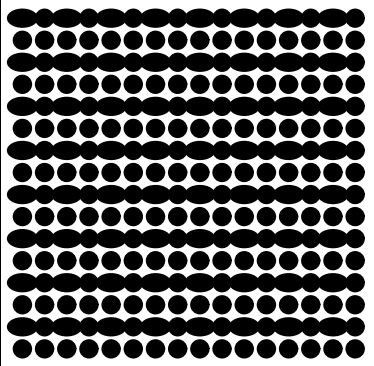

(c) Hexagonal Dispersed Dither level i+2
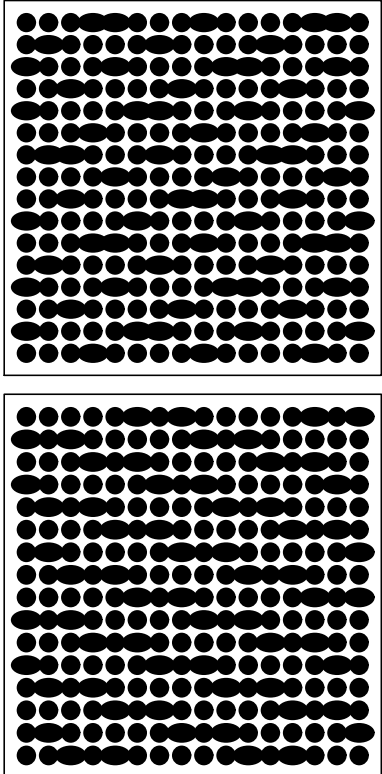

Figure 3. Breaking the horizontal bands produced by elliptic dot shapes with distributions of dither thresholds according to the tiles shown in Fig. 2a and $2 b$. 


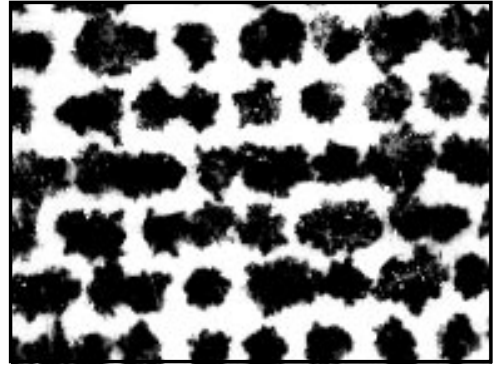

Figure 4. Enlargement of a halftone pattern printed by a real multi-level inkjet printer on plain paper

(a) Bayer Dispersed Dither level i+2

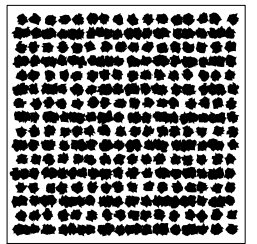

(b) Parallelogram Dispersed Dither level i+2

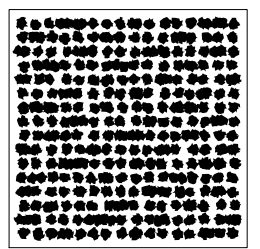
Dispersed Dither level $\mathrm{i}+2$
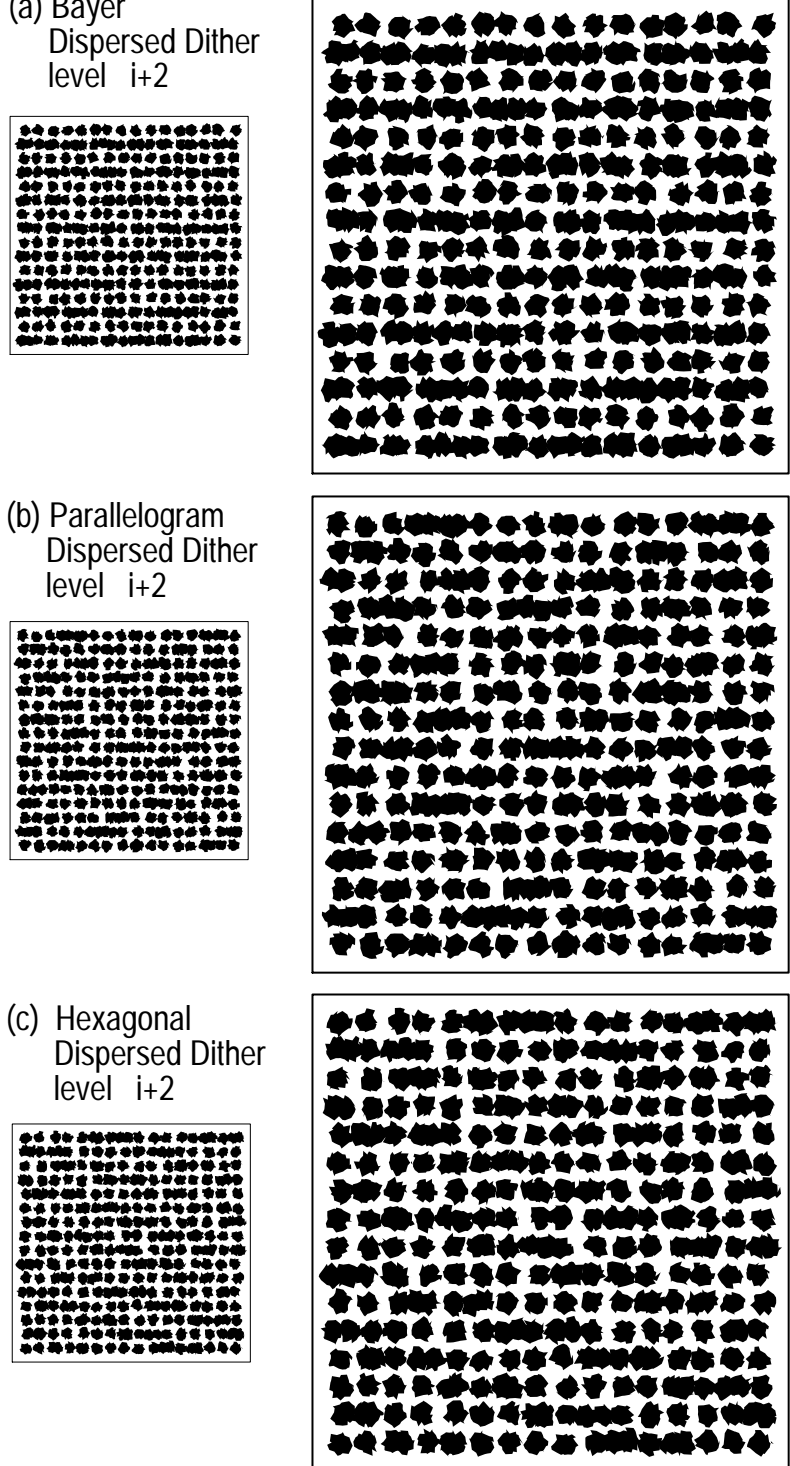

\section{CONCLUSIONS}

Variable dot size inkjet printers at moderate cost will soon be available on the market. Due to the relatively large size of single droplets, halftoning algorithms are still needed. However, since halftoning occurs between the basic levels attainable by printing one, two or several droplets at the same position, artefacts are less visible than in equal resolution bilevel printers. When dithering algorithms are used for the halftoning task, the dither threshold tiles should have oblique orientations so as to make the halftoning artifacts less visible. They should be designed so as to break up the inherent artifacts of variable dot size printers, such as for example continuous lines made up of elongated elliptic dots.

\section{REFERENCES}

[1] T. Mitsa, K.J. Parker, "Digital halftoning technique using a blue-noise mask, J.Opt.Soc.Am. A 9(11), 1992, 1920-1929.

[2] V. Ostromoukhov, R.D. Hersch, I. Amidror, "Rotated Dispersed Dither: a New Technique for Digital Halftoning", ACM Siggraph'94, Conference Graphics Proceedings, Annual Conference Series, 1994, 123-130.

[3] R. Eschbach, "Reduction of artifacts in error diffusion by means of input-dependent weights", Journal of Electronic Imaging, Vol.2 , No. 4, Octobre 1993, 352-358.

[4] R.L. Miller, R.A. Morton (inventors), Image Processor with Smooth Transitioning between Dither and Diffusion Processes, US Patent 5'014'333, issued May 7, 1991, filed Jul. 21, 1988.

[5] B.E. Bayer, "An Optimum Method for Two-Level Rendition of Continuous-Tone Pictures", IEEE 1973 International Conf. on Communications, Vol. 1, 26.11 - 26-15

[6] R. Ulichney, Digital Halftoning, The MIT Press, Cambridge, Mass., 1987.

[7] J.F. Jarvis, C.N. Judice, W.H. Ninke, "A Survey of Techniques for the Display of Continuous-Tone Pictures on Bilevel Displays, " Computer Graphics and Image Processing, Vol 5, 1976, 13-40.

[8] Peter Stucki, "MECCA - A multiple error correcting computation algorithm for bilevel image hardcopy reproduction, Research Report RZ1060, IBM Res. Lab., Zurich, Switzerland, 1981.

[9] V. Ostromoukhov, Reproduction couleur par trames irrégulières et semi-régulières, $\mathrm{PhD}$ thesis no 1330 , EPFL, Lausanne, 1995.

Figure 5. Simulation of real impacts 


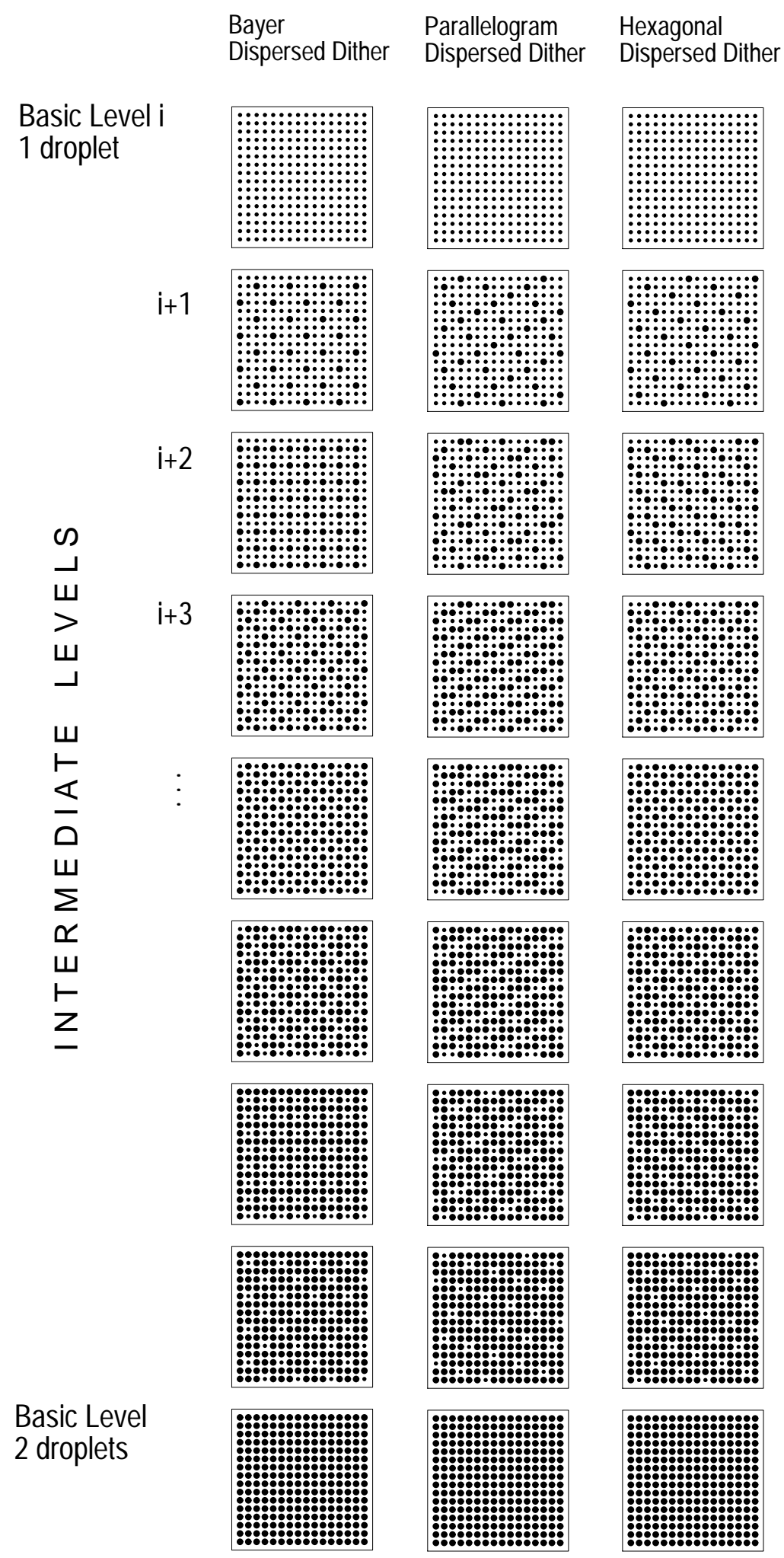

Figure 6. Intermediate intensity levels produced by dither tile based multi-level printing; the parallelogram dither corresponds to the tile shown in Fig. 2a and the hexagonal dither to the tile shown in Fig. $2 b$. 